PROCEEDINGS OF THE

AMERICAN MATHEMATICAL SOCIETY

Volume 139, Number 7, July 2011, Pages 2485-2496

S 0002-9939(2011)10794-9

Article electronically published on January 4, 2011

\title{
ERGODIC ACTIONS OF CONVERGENT FUCHSIAN GROUPS ON QUOTIENTS OF THE NONCOMMUTATIVE HARDY ALGEBRAS
}

\author{
ALVARO ARIAS AND FRÉDÉRIC LATRÉMOLIÈRE \\ (Communicated by Marius Junge)
}

\begin{abstract}
We establish that particular quotients of the noncommutative Hardy algebras carry ergodic actions of convergent discrete subgroups of the group $\mathrm{SU}(n, 1)$ of automorphisms of the unit ball in $\mathbb{C}^{n}$. To do so, we provide a mean to compute the spectra of quotients of noncommutative Hardy algebra and characterize their automorphisms in terms of biholomorphic maps of the unit ball in $\mathbb{C}^{n}$.
\end{abstract}

We establish that given any discrete subgroup $\Gamma$ of $\mathrm{SU}(n, 1)$ such that the orbit of 0 for the action of $\Gamma$ on the open unit ball $\mathbb{B}_{n}$ of $\mathbb{C}^{n}$ satisfies the Blaschke condition

$$
\sum_{\gamma \in \Gamma}\left(1-\|\gamma(0)\|_{\mathbb{C}^{n}}\right)<\infty
$$

there exists a quotient algebra of the noncommutative Hardy algebra $F_{n}^{\infty}$ whose group of weak* continuous automorphisms is the stabilizer $\underline{\Gamma}$ of the orbit of 0 for $\Gamma$ in $\mathbb{B}_{n}$. Moreover, $\Gamma$ acts ergodically on this quotient algebra. Our methods rely heavily on the theory of analytic functions in several variables.

The noncommutative Hardy algebra $F_{n}^{\infty}$ is the weak-operator closure of the left regular representation of the free semigroup on $n$ generators, and it is a noncommutative analogue of the Hardy algebra $H^{\infty}\left(\mathbb{B}_{1}\right)$ [7]. Our motivation for this study is to explore the very rich ideal structure of $F_{n}^{\infty}$, as well as the structure of automorphism groups of non-self-adjoint operator algebras. This paper is based upon the remarkable result of Davidson and Pitts [3, Theorem 4.11], where the group of completely contractive automorphisms of $F_{n}^{\infty}$ is $\mathrm{SU}(n, 1)$, i.e. the same group as the group of biholomorphic maps from $\mathbb{B}_{n}$ into itself [3, Lemma 4.9]. See also [13] and [11] for other approaches to this fact. We recall that $\mathrm{SU}(n, 1)$ is the Lie group of $(n+1) \times(n+1)$ matrices of bilinear maps on $\mathbb{C}^{n}$ preserving the canonical sesquilinear form of signature $(1, n)$.

Let us recall the definition of $F_{n}^{\infty}$ 7. Let $n \in \mathbb{N}$ with $n>0$. The full Fock space $F^{2}\left(\mathbb{C}^{n}\right)$ is the completion of

$$
\bigoplus_{k \in \mathbb{N}}\left(\mathbb{C}^{n}\right)^{\otimes k}=\mathbb{C} \oplus \mathbb{C}^{n} \oplus\left(\mathbb{C}^{n} \otimes \mathbb{C}^{n}\right) \oplus\left(\mathbb{C}^{n} \otimes \mathbb{C}^{n} \otimes \mathbb{C}^{n}\right) \oplus \cdots
$$

Received by the editors March 2, 2010 and, in revised form, June 30, 2010.

2010 Mathematics Subject Classification. Primary 47L15, 47L55; Secondary 32M05.

Key words and phrases. Noncommutative Hardy algebras, Fock spaces, Fuchsian group, Mobius transformations, automorphisms of operator algebras.

(C)2011 American Mathematical Society Reverts to public domain 28 years from publication 
for the Hilbert norm associated to the inner product $\langle.,$.$\rangle defined on elementary$ tensors by

$$
\left\langle\xi_{0} \otimes \cdots \otimes \xi_{m}, \zeta_{0} \otimes \cdots \otimes \zeta_{k}\right\rangle=\left\{\begin{array}{c}
0 \text { if } m \neq k \\
\prod_{j=0}^{n}\left\langle\xi_{j}, \zeta_{j}\right\rangle_{\mathbb{C}^{n}} \text { otherwise }
\end{array}\right.
$$

where $\langle., .\rangle_{\mathbb{C}^{n}}$ is the canonical inner product on $\mathbb{C}^{n}$. Let $\left\{e_{1}, \ldots, e_{n}\right\}$ be the canonical basis of $\mathbb{C}^{n}$ and, naturally, let 1 be the canonical basis of $\mathbb{C}$. We define, for each $j \in\{1, \ldots, n\}$, an operator $S_{j}$ on $F_{n}^{2}\left(\mathbb{C}^{n}\right)$ by

$$
S_{j}\left(e_{i_{1}} \otimes \cdots \otimes e_{i_{m}}\right)=e_{j} \otimes e_{i_{1}} \otimes \cdots \otimes e_{i_{m}} \text { and } S_{j}(1)=e_{j} .
$$

The operators $S_{1}, \ldots, S_{n}$ are called the left creation operators, and we observe that $\sum_{j=1}^{n} S_{j} S_{j}^{*} \leq 1$, i.e. $\left[S_{1} \cdots S_{n}\right]$ is a row contraction. The weak-operator-topology closure of the algebra generated by $\left\{1, S_{1}, \ldots, S_{n}\right\}$ is the noncommutative Hardy algebra $F_{n}^{\infty}$. The fundamental property of $F_{n}^{\infty}$ is that, given any separable Hilbert space $\mathcal{H}$ and any $n$-tuple $T=\left(T_{1}, \ldots, T_{n}\right)$ of operators on $\mathcal{H}$ such that $\sum_{j=1}^{n} T_{j} T_{j}^{*}<$ 1 , there exists a unique completely contractive algebra homomorphism $\pi_{T}$ from $F_{n}^{\infty}$ into the algebra of bounded linear operators on $\mathcal{H}$ such that $\pi_{T}\left(S_{j}\right)=T_{j}$ for $j=1, \ldots, n$, and we note that this map is weak* continuous. This property was established by Popescu in 7 ] and in [9] using a noncommutative generalization of the Poisson transform. As a matter of notation, we will write $\varphi\left(T_{1}, \ldots, T_{n}\right)$ for the operator $\pi_{T}(\varphi)$ whenever $\varphi \in F_{n}^{\infty}$. The algebra $F_{n}^{\infty}$ plays a very important role in interpolation theory, among other matters, and many results valid for the Hardy algebra $H^{\infty}\left(\mathbb{B}_{1}\right)$ can be extended to $F_{n}^{\infty}$.

The Banach space $F_{n}^{\infty}$ is a dual space and thus can be endowed with the corresponding weak* topology, which agrees with the restriction of the weak-operator topology to $F_{n}^{\infty}$. In this paper, we will refer to this topology on $F_{n}^{\infty}$ as the weak* topology on $F_{n}^{\infty}$ [4]. We also note that Davidson and Pitts use the notation $\mathcal{L}_{n}$ for $F_{n}^{\infty}$, but we shall prefer Popescu's notation to emphasize the connection with the Hardy algebra.

In this paper, we define the spectrum of an operator algebra as the space of all weak* continuous scalar-valued algebra homomorphisms, endowed with the $\sigma\left(F_{n}^{\infty *}, F_{n}^{\infty}\right)$ topology (i.e. the weak* topology on the dual of $\left.F_{n}^{\infty}\right)$. As a consequence of the fundamental property of $F_{n}^{\infty}$, we note that the spectrum of $F_{n}^{\infty}$ consists exactly of the maps $\pi: F_{n}^{\infty} \longrightarrow \mathbb{C}$ such that $\sum_{j=1}^{n}\left|\pi\left(S_{j}\right)\right|^{2}<1$, and one checks that indeed the spectrum is homeomorphic to $\mathbb{B}_{n}[8$.

While we use Popescu's $F_{n}^{\infty}$ in this paper, one should observe that all our quotient algebras are in fact commutative. Indeed, we quotient $F_{n}^{\infty}$ by intersections of kernels of scalar-valued algebra homomorphisms, which always contain the commutator ideal of $F_{n}^{\infty}$. Hence, all our constructions factor through the multiplier algebra of the symmetric Fock space, as was studied in [2].

This paper is organized as follows. The first section provides a functional mean to compute the pseudohyperbolic metric on $\mathbb{B}_{n}$ which will prove important for our purpose. We then compute the spectrum of a large class of quotient algebras of $F_{n}^{\infty}$. We then conclude that some of these quotients admit a discrete subgroup of $S U(n, 1)$ as their group of automorphism. 
Last, as a matter of notation, we will denote the norm on a Banach space $E$ by $\|\cdot\|_{E}$, where there is no ambiguity. The norm $\|\cdot\|_{\mathbb{C}^{n}}$ is the canonical hermitian norm.

\section{Spectra of Quotients of $F_{n}^{\infty}$}

This section addresses the duality between the process of associating an ideal of $F_{n}^{\infty}$ to a subset of the open unit ball $\mathbb{B}_{n}$ of $\mathbb{C}^{n}$ and the computation of the spectrum of a quotient of $F_{n}^{\infty}$. To this end, we first observe that since $F_{n}^{\infty}$ is a dual space, given any weak ${ }^{*}$ closed ideal $\mathcal{J}$ in $F_{n}^{\infty}$, the space $F_{n}^{\infty} / \mathcal{J}$ is dual to the polar $\mathcal{J}^{\circ}$ of $\mathcal{J}$ in the predual of $F_{n}^{\infty}$, and thus can be endowed with the weak* topology. We can thus define:

Definition 1.1. Let $\mathcal{J}$ be a weak ${ }^{*}$ closed two-sided ideal in $F_{n}^{\infty}$. A weak ${ }^{*}$ continuous unital algebra homomorphism from $F_{n}^{\infty} / \mathcal{J}$ to $\mathbb{C}$ is called a weak ${ }^{*}$ scalar representation of $F_{n}^{\infty} / \mathcal{J}$, or when no confusion may arise, a representation of $F_{n}^{\infty} / \mathcal{J}$.

Definition 1.2. Let $\mathcal{J}$ be a weak* closed two-sided ideal in $F_{n}^{\infty}$. We define the spectrum $\Sigma\left(F_{n}^{\infty} / \mathcal{J}\right)$ of $F_{n}^{\infty} / \mathcal{J}$ as the set of all weak ${ }^{*}$ scalar representations of $F_{n}^{\infty} / \mathcal{J}$, endowed with the weak* topology of the dual of $F_{n}^{\infty} / \mathcal{J}$.

Let us note that this definition involves two distinct topologies. As a set, $\Sigma\left(F_{n}^{\infty} / \mathcal{J}\right)$ is defined as the collection of scalar-valued unital algebra homorphisms of $F_{n}^{\infty}$ which are continuous for the weak ${ }^{*}$ topology of $F_{n}^{\infty} / \mathcal{J}$ seen as the dual space of $\mathcal{J}^{\circ}$. On the other hand, the topology on $\Sigma\left(F_{n}^{\infty} / \mathcal{J}\right)$ is the restriction of the weak ${ }^{*}$ topology of the dual $\left(F_{n}^{\infty} / \mathcal{J}\right)^{*}$ of $F_{n}^{\infty} / \mathcal{J}$.

Now, by [7], for any $\lambda=\left(\lambda_{1}, \ldots, \lambda_{n}\right) \in \mathbb{B}_{n}$ we can define a unique representation $\pi_{\lambda}$ of $F_{n}^{\infty}$ such that $\pi_{\lambda}\left(S_{j}\right)=\lambda_{j}$ with $j=1, \ldots, n$. Moreover, by [7] the map $\lambda \in \mathbb{B}_{n} \mapsto \pi_{\lambda}$ is a homeomorphism. Given any $\varphi \in F_{n}^{\infty}$, we denote $\pi_{\lambda}(\varphi)$ by $\varphi(\lambda)$, thus using duality to see elements of $F_{n}^{\infty}$ as functions on $\mathbb{B}_{n}$. With this notation, we have the following standard result:

Proposition 1.3. Let $\mathcal{J}$ be a weak ${ }^{*}$ closed two-sided ideal in $F_{n}^{\infty}$. Then

$$
\Sigma\left(F_{n}^{\infty} / \mathcal{J}\right)=\left\{z \in \mathbb{B}_{n}: \forall \varphi \in \mathcal{J} \quad \varphi(z)=0\right\}
$$

Proof. The canonical projection $q: F_{n}^{\infty} \rightarrow F_{n}^{\infty} / \mathcal{J}$ is weak ${ }^{*}$ continuous, and thus if $\pi$ is a weak ${ }^{*}$ continuous algebra homomorphism from $F_{n}^{\infty} / \mathcal{J}$ to $\mathbb{C}$, then $\pi \circ q$ is in the spectrum of $F_{n}^{\infty}$. Conversely, if $\pi$ is in the spectrum of $F_{n}^{\infty}$ such that $\pi(\mathcal{J})=\{0\}$, then $\pi$ defines an element of the spectrum of $F_{n}^{\infty} / \mathcal{J}$. We thus have proven that the spectrum of $F_{n}^{\infty} / \mathcal{J}$ is given by $\left\{z \in \mathbb{B}_{n}: \forall \varphi \in F_{n}^{\infty} \quad \varphi(z)=0\right\}$.

We observe that, by Proposition 1.3. if $\varphi \in F_{n}^{\infty} / \mathcal{J}$, where $\mathcal{J}$ is a weak ${ }^{*}$ closed two-sided ideal in $F_{n}^{\infty}$ and $\lambda \in \Sigma\left(F_{n}^{\infty} / \mathcal{J}\right)$, then $\varphi(\lambda)$ is well-defined as the common value of $\psi(\lambda)$ for $\psi \in F_{n}^{\infty}$ such that $\psi+\mathcal{J}=\varphi$ (where $\psi+\mathcal{J}$ is the class of $\psi$ in $\left.F_{n}^{\infty} / \mathcal{J}\right)$. Now, we can use this function representation of $F_{n}^{\infty} / \mathcal{J}$ on its spectrum to associate a natural holomorphic map to any automorphism $\Phi$ of $F_{n}^{\infty} / \mathcal{J}$. Indeed, if $\pi$ is a weak* scalar representation of $F_{n}^{\infty} / \mathcal{J}$, then so is $\pi \circ \Phi$ for all weak* continuous automorphisms of $F_{n}^{\infty} / \mathcal{J}$. Hence, we can define the following:

Definition 1.4. Let $\mathcal{J}$ be a weak* closed two-sided ideal in $F_{n}^{\infty}$. Let $\Phi$ be a weak* continuous automorphism of $F_{n}^{\infty} / \mathcal{J}$. The dual map $\widehat{\Phi}$ of $\Phi$ is defined for all $\lambda \in \Sigma\left(F_{n}^{\infty} / \mathcal{J}\right)$ by

$$
\widehat{\Phi}(\lambda)=\left(\Phi^{-1}\left(S_{1}\right)(\lambda), \ldots, \Phi^{-1}\left(S_{1}\right)(\lambda)\right)
$$


Definition 1.5. The group of weak* completely isometric continuous automorphisms of $F_{n}^{\infty} / \mathcal{J}$ is denoted by $\operatorname{Aut}\left(F_{n}^{\infty} / \mathcal{J}\right)$.

It is an immediate observation that the map $\Phi \in \operatorname{Aut}\left(F_{n}^{\infty} / \mathcal{J}\right) \mapsto \widehat{\Phi}$ is a group homomorphism to the group of homeomorphisms of $\Sigma\left(F_{n}^{\infty} / \mathcal{J}\right)$ (in particular, mapping the identity to the identity) such that

$$
\forall \varphi \in F_{n}^{\infty} / \mathcal{J} \quad \forall \lambda \in \Sigma\left(F_{n}^{\infty} / \mathcal{J}\right) \quad \Phi(\varphi)(\lambda)=\varphi\left(\widehat{\Phi}^{-1}(\lambda)\right) .
$$

Although, for the general completely contractive homomorphism $\Phi$, one would be inclined to define the dual of $\Phi$ as $\Phi^{\prime}: \lambda \in \Sigma\left(F_{n}^{\infty} / \mathcal{J}\right) \mapsto\left(\Phi\left(S_{j}\right)(\lambda)\right)_{j=1, \ldots, n}$. The resulting map restricted to automorphisms would be valued at the opposite end of the group of homeomorphisms of $\Sigma\left(F_{n}^{\infty} / \mathcal{J}\right)$, which would be inconvenient. Hence, we adopt our modified definition in this paper to obtain a group morphism. This is the same definition as in [3] when $\mathcal{J}=\{0\}$.

Although it is a priori only a topological space homeomorphic to $\mathbb{B}_{n}$, the spectrum $\Sigma\left(F_{n}^{\infty}\right)$ is in fact endowed with a complex structure via its relation with $F_{n}^{\infty}$ [7]: the maps $\varphi$ defined by elements of $F_{n}^{\infty}$ on $\mathbb{B}_{n}$ are holomorphic, and so are the dual maps of automorphisms [3, Theorem 4.11]. These results can be extended to more general complex domains 10. This allows us to use techniques from the theory of analytic functions in several complex variables, as in 1.

Our main focus in this section consists of the following two related notions:

Definition 1.6. Let $\Delta \subseteq \mathbb{B}_{n}$. The Nevanlinna ideal for $\Delta$ in $F_{n}^{\infty}$ is

$$
\mathcal{N}_{\Delta}=\left\{\varphi \in F_{n}^{\infty}: \forall z \in \Delta \quad \varphi(z)=0\right\},
$$

and the quotient of $F_{n}^{\infty}$ localized at $\Delta$ is $F_{n, \Delta}^{\infty}=F_{n}^{\infty} / \mathcal{N}_{\Delta}$.

Definition 1.7. Let $\Delta \subseteq \mathbb{B}_{n}$. The spectrum of $F_{n, \Delta}^{\infty}$ is called the spectral closure of $\Delta$ and is denoted by $\bar{\Delta}^{\Sigma}$.

By Proposition 1.3 , given $\Delta \subseteq \mathbb{B}_{n}$, we always have $\Delta \subseteq \bar{\Delta}^{\Sigma}$. In general, we can have $\Delta \subsetneq \bar{\Delta}^{\Sigma}$, as shown for instance when $\Delta=\left\{0, \frac{1}{n}: n \in \mathbb{N}, n>0\right\} \subset \mathbb{B}_{1}$, since there is no nonzero holomorphic function which is null on $\Delta$, so $\bar{\Delta}^{\Sigma}=\mathbb{B}_{1}$. In other words, the main issue when relating $\Delta \subseteq \mathbb{B}_{n}$ with $\bar{\Delta}^{\Sigma}$ is that the Nevanlinna ideal $\mathcal{N}_{\Delta}$ may be null. It is well-known [6, 9.1.4, 9.1.5] that, for $n=1$, a set $\Delta$ is the zero set for some holomorphic function if and only if it satisfies the Blaschke condition, i.e. $\Delta=\left\{\lambda_{j}: j \in \mathbb{N}\right\}$ with $\sum_{j=0}^{\infty}\left(1-\left|\lambda_{j}\right|\right)<\infty$. Under this condition, the Blachke product associated to $\Delta$ is a holomorphic function which is zero exactly on $\Delta$. Unfortunately, such a result does not hold in higher dimension [5, Ch. 9]. However, we shall now prove that the Blaschke condition is still sufficient to ensure that $\Delta=\bar{\Delta}^{\Sigma}$ in $\mathbb{B}_{n}$.

To this end, we shall use the geometry of $\mathbb{B}_{n}$ by providing a formula connecting the Poincaré pseudohyperbolic metric on $\mathbb{B}_{n}$ with the unit ball of $F_{n}^{\infty}$. As a tool for our proof, we will use the following lemma, which is a special case of [12, Theorem 8.1.4] and which will find a role in the next section as well. We include a proof of this lemma for the reader's convenience.

Lemma 1.8. Let $\varphi$ be a holomorphic function from $\mathbb{B}_{n}$ to $\mathbb{B}_{k}$ for some nonzero natural $k$ and such that $\varphi(0)=0$. Then for all $z \in \mathbb{B}_{n}$ we have $\|\varphi(z)\|_{\mathbb{C}^{k}} \leq\|z\|_{\mathbb{C}^{n}}$. 
Proof. Let $z \in \mathbb{B}_{n}$. Since the result is trivial for $z=0$, we shall assume $z \neq 0$. Let $\theta: \mathbb{C}^{k} \rightarrow \mathbb{C}$ be a linear functional of norm 1 (for the dual norm to the canonical Hermitian norm on $\left.\mathbb{C}^{k}\right)$ such that $|\theta \circ \varphi(z)|=\|\varphi(z)\|_{\mathbb{C}^{k}}$. We define the map $\varphi_{z}^{\theta}: \mathbb{B}_{1} \longrightarrow \mathbb{B}_{1}$ by $\varphi_{z}^{\theta}(t)=\theta \circ \varphi\left(t \frac{z}{\|z\|_{\mathbb{C}^{n}}}\right)$ for $t \in \mathbb{B}_{1}$. By construction, $\varphi_{z}^{\theta}$ is holomorphic from the unit disk into itself and $\varphi_{z}^{\theta}(0)=0$. Hence, by the Schwarz lemma, we have for all $t \in \mathbb{B}_{1}$ that $\left|\varphi_{z}^{\theta}(t)\right| \leq|t|$. In particular,

$$
\|z\|_{\mathbb{C}^{n}} \geq\left|\varphi_{z}^{\theta}(\|z\|)\right|=|\theta \circ \varphi(z)|=\|\varphi(z)\|_{\mathbb{C}^{k}} .
$$

Hence our lemma is proven.

Poincaré's pseudohyperbolic metric on the open unit ball $\mathbb{B}_{n}$ of $\mathbb{C}^{n}$ between two points $z$ and $w$ can be defined as the Euclidean distance $\rho$ between 0 and the image of $w$ by any biholomorphic function of the ball which maps $z$ to 0 . As customary in complex analysis, we will refer to biholomorphic maps of $\mathbb{B}_{n}$ onto itself as automorphisms of $\mathbb{B}_{n}$. We show that it is also possible to compute this distance by using $F_{n}^{\infty}$.

Proposition 1.9. Let $\rho$ be the Poincaré pseudohyperbolic metric on $\mathbb{B}_{n}$. For any $z, w \in \mathbb{B}_{n}$ we have

$$
\rho(z, w)=\max \left\{|\varphi(z)|: \varphi \in F_{n}^{\infty} \text { with }\|\varphi\|_{F_{n}^{\infty}} \leq 1 \text { and } \varphi(w)=0\right\} .
$$

Proof. We define for all $z, w \in \mathbb{B}_{n}$ the quantity

$$
\eta(z, w)=\sup \left\{|\varphi(z)|: \varphi \in F_{n}^{\infty} \text { with }\|\varphi\|_{F_{n}^{\infty}} \leq 1 \text { and } \varphi(w)=0\right\} .
$$

We wish to show that $\eta=\rho$ and that the supremum defining $\eta$ is, in fact, reached.

First, we prove that $\eta$ is invariant under the action of $\operatorname{SU}(n, 1)$ on $\mathbb{B}_{n}$. Let $z, w \in \mathbb{B}_{n}$ and let $\widehat{\Phi}$ be an automorphism of $\mathbb{B}_{n}$. There exists by [3] a unique automorphism $\Phi$ of $F_{n}^{\infty}$ such that, for all $\omega \in \mathbb{B}_{n}$ and $\varphi \in F_{n}^{\infty}$, we have

$$
\Phi^{-1}(\varphi)(\omega)=\varphi(\widehat{\Phi}(\omega)) .
$$

(Of course, we could denote this automorphism of $F_{n}^{\infty}$ by $\Phi$ rather than $\Phi^{-1}$, but we prefer to keep the notation for dual map consistent in this paper.)

As an automorphism of $F_{n}^{\infty}$ it is an isometry and thus maps the unit ball of $F_{n}^{\infty}$ onto itself. Thus:

$$
\begin{aligned}
& \eta(\widehat{\Phi}(z), \widehat{\Phi}(w))=\sup \left\{\begin{array}{l|l}
|\varphi(\widehat{\Phi}(z))| & \begin{array}{l}
\varphi \in F_{n}^{\infty}, \\
\|\varphi\|_{F_{n}}^{\infty} \leq 1, \\
\varphi(\widehat{\Phi}(w))=0
\end{array}
\end{array}\right\} \\
& =\sup \left\{\begin{array}{l|l}
\left|\Phi^{-1}(\varphi)(z)\right| & \begin{array}{l}
\varphi \in F_{n}^{\infty}, \\
\left\|\Phi^{-1}(\varphi)\right\|_{F_{n}^{\infty}} \leq 1, \\
\Phi^{-1}(\varphi)(w)=0
\end{array}
\end{array}\right\} \\
& =\sup \left\{\begin{array}{l|l}
|\psi(z)| & \begin{array}{l}
\psi \in F_{n}^{\infty}, \\
\|\psi\|_{F_{n}^{\infty}} \leq 1, \\
\psi(w)^{2}=0
\end{array}
\end{array}\right\}=\eta(z, w) .
\end{aligned}
$$

In particular, $\eta(z, w)=\eta(w, z)$. as there exists an automorphism of $\mathbb{B}_{n}$ which maps $z$ to $w$ and vice versa. Thus, it is enough to prove that, for any $z \in \mathbb{B}_{n}$, we have 
$\rho(0, z)=\|z\|_{\mathbb{C}^{n}}$. This would suffice to show that $\eta$ is the Poincaré pseudohyperbolic metric $\rho$ on $\mathbb{B}_{n}$.

Let us fix $z \in \mathbb{B}_{n}$. Since $\eta(0,0)=0$, we may as well assume $z \neq 0$. Let $\varphi \in F_{n}^{\infty}$ such that $\|\varphi\|_{F_{n}^{\infty}} \leq 1$ and $\varphi(0)=0$. By Lemma 1.8, we have $|\varphi(z)| \leq\|z\|_{\mathbb{C}^{n}}$ so $\eta(0, z)$, which is the supremum of $|\varphi(z)|$ for $\varphi \in F_{n}^{\infty}$ with $\|\varphi\|_{F_{n}^{\infty}} \leq 1$ and $\varphi(0)=0$, is bounded above by $\|z\|_{\mathbb{C}^{n}}$. On the other hand, observe that for any $a_{1}, \ldots, a_{n} \in \mathbb{C}^{n}$, if $\varphi=\sum_{j=1}^{n} a_{j} S_{j}$, then the norm of $\varphi$ is $\left\|\varphi^{*} \varphi\right\|_{\mathcal{B}\left(F_{n}^{2}\right)}^{\frac{1}{2}}$, which equals $\sqrt[2]{\sum_{j=1}^{n}\left|a_{j}\right|^{2}}$ since $S_{j}^{*} S_{k}=\delta_{j}^{k} 1$. If we write $z=\left(z_{1}, \ldots, z_{n}\right)$, then choosing $\varphi_{z}=\sum_{j=1}^{n} \frac{z_{i}}{\|z\|_{\mathbb{C}^{n}}} S_{i}$ we see that $\varphi_{z} \in F_{n}^{\infty}$ with $\varphi_{z}(0)=0$ and $\left|\varphi_{z}(z)\right|=\|z\|_{\mathbb{C}^{n}}$. So $\|z\|_{\mathbb{C}^{n}} \leq \eta(0, z)$ as desired. We conclude that $\eta(0, z)=\|z\|_{\mathbb{C}^{n}}$, and this supremum is reached at $\varphi_{z}$.

We can now prove that the Blaschke condition is sufficient for a subset $\Delta$ of $\mathbb{B}_{n}$ to equal its spectral closure. We start with the following lemma, which uses an important estimate from the theory of functions on $\mathbb{B}_{n}$.

Lemma 1.10. Let $\left\{\lambda_{j}: j \in \mathbb{N}\right\} \subseteq \mathbb{B}_{n}$. Let $\varphi$ be an automorphism of $\mathbb{B}_{n}$ such that $\varphi(0) \neq 0$. Then

$$
\sum_{j=0}^{\infty}\left(1-\left\|\lambda_{j}\right\|_{\mathbb{C}^{n}}\right)<\infty \Longleftrightarrow \sum_{j=0}^{\infty}\left(1-\left\|\varphi\left(\lambda_{j}\right)\right\|_{\mathbb{C}^{n}}\right)<\infty .
$$

Proof. We denote $\|\cdot\|_{\mathbb{C}^{n}}$ by $\|\cdot\|$ in this proof. Let $a=\varphi^{-1}(0)$ and note that $a \neq 0$ by assumption. Using [12, Theorem 2.2.2, p. 26], we have for all $z \in \mathbb{B}_{n}$ :

$$
1-\|\varphi(z)\|=\frac{\left(1-\|a\|^{2}\right)(1+\|z\|)}{(1+\|\varphi(z)\|)|1-\langle a, z\rangle|}(1-\|z\|),
$$

where $\langle.,$.$\rangle is the canonical inner product in \mathbb{C}^{n}$. Therefore, for all $z \in \mathbb{B}_{n}$, we have

$$
0<\frac{1-\|a\|^{2}}{2} \leq \frac{\left(1-\|a\|^{2}\right)(1+\|z\|)}{(1+\|\varphi(z)\|)|1-\langle a, z\rangle|} \leq \frac{2}{1-\|a\|} .
$$

The result follows.

Theorem 1.11. Let $\Delta=\left\{\lambda_{j}: j \in \mathbb{N}\right\} \subseteq \mathbb{B}_{n}$. If $\sum_{j=0}^{\infty}\left(1-\left\|\lambda_{j}\right\|_{\mathbb{C}^{n}}\right)<\infty$, then the weak $^{*}$ spectrum $\Sigma\left(F_{n, \Delta}^{\infty}\right)$ of $F_{n, \Delta}^{\infty}=F_{n}^{\infty} / \mathcal{N}_{\Delta}$ is $\Delta$.

Proof. Assume we are given $\Delta=\left\{\lambda_{j}: j \in \mathbb{N}\right\} \subseteq \mathbb{B}_{n}$ with $\sum_{j=0}^{\infty}\left(1-\left\|\lambda_{j}\right\|_{\mathbb{C}^{n}}\right)<\infty$. Let $z \in \mathbb{B}_{n} \backslash \Delta$. We wish to show that there exists $\varphi \in \mathcal{N}_{\Delta}$ such that $\varphi(z) \neq 0$. Let $\widehat{\Phi}$ be an automorphism of $\mathbb{B}_{n}$ which maps $z$ to 0 . By Lemma 1.9] for each $j \in \mathbb{N}$ there exists $\varphi_{j} \in F_{n}^{\infty}$ such that $\left\|\varphi_{j}\right\|_{\mathbb{C}^{n}} \leq 1, \varphi_{j}\left(\widehat{\Phi}\left(\lambda_{j}\right)\right)=0$ and $\left\|\widehat{\Phi}\left(\lambda_{j}\right)\right\|_{\mathbb{C}^{n}}=\varphi_{j}(0)$. Fix $j \in \mathbb{N}$. We define the element $\psi_{j}=\prod_{k=0}^{j} \varphi_{k} \in F_{n}^{\infty}$. By construction, we have $\left\|\psi_{j}\right\|_{F_{n}^{\infty}} \leq 1$, as well as $\psi_{j}(0)=\prod_{k=0}^{j}\left\|\widehat{\Phi}\left(\lambda_{j}\right)\right\|_{\mathbb{C}^{n}}$ and $\psi_{j}\left(\widehat{\Phi}\left(\lambda_{k}\right)\right)=0$ for $k \in\{0, \ldots, j\}$. Now, the unit ball of $F_{n}^{\infty}$ is weak* compact, so we can extract a subsequence of $\left(\psi_{j}\right)_{j \in \mathbb{N}}$ which converges in the weak* topology to some $\psi \in F_{n}^{\infty}$. 
We recall that by definition, the notation $\psi(\mu)$ refers to $\pi_{\mu}(\psi)$, where $\pi_{\mu}$ is the unique unital weak* continuous algebra homomorphism from $F_{n}^{\infty}$ to $\mathbb{C}$ mapping the canonical generators $S_{1}, \ldots, S_{n}$ of $F_{n}^{\infty}$ to $\mu_{1}, \ldots, \mu_{n}$ with $\mu=\left(\mu_{1}, \ldots, \mu_{n}\right)$. Hence, by continuity, we have in particular that since $\sum_{j=0}^{\infty}\left(1-\left\|\lambda_{j}\right\|_{\mathbb{C}^{n}}\right)<\infty$, using Lemma 1.10, we have $\sum_{j=0}^{\infty}\left(1-\left\|\widehat{\Phi}\left(\lambda_{j}\right)\right\|_{\mathbb{C}^{n}}\right)<\infty$ and thus

$$
\psi(0)=\prod_{j=0}^{\infty}\left\|\widehat{\Phi}\left(\lambda_{j}\right)\right\|_{\mathbb{C}^{n}}>0 .
$$

Hence $\psi(0) \neq 0$, while $\psi\left(\widehat{\Phi}\left(\lambda_{j}\right)\right)=0$ by construction as well. Thus, if $\Phi$ is the automorphism of $F_{n}^{\infty}$ whose dual map is $\widehat{\Phi}$ and if we set $\varphi=\Phi^{-1}(\psi)$, we see that $\varphi \in \mathcal{N}_{\Delta}$ while $\varphi(z) \neq 0$, so $z \notin \bar{\Delta}^{\Sigma}$. Thus $\bar{\Delta}^{\Sigma} \subseteq \Delta$. Since the reverse inclusion is Proposition 1.3, our theorem is established.

\section{Automorphism groups of Quotients of $F_{n}^{\infty}$}

This section establishes that, under a natural condition of convergence, it is possible to choose many discrete subgroups of $\mathrm{SU}(n, 1)$ as full automorphism groups of some operator algebras obtained as quotients of $F_{n}^{\infty}$. We shall call any biholomorphic from $\mathbb{B}_{n}$ onto $\mathbb{B}_{n}$ an automorphism of $\mathbb{B}_{n}$. The group of automorphisms of $\mathbb{B}_{n}$ is denoted by Aut $\left(\mathbb{B}_{n}\right)$.

We start with an easy consequence of [3]:

Lemma 2.1. Let $\Delta \subseteq \mathbb{B}_{n}$. Let $\phi$ be an automorphism of $\mathbb{B}_{n}$ such that $\phi\left(\bar{\Delta}^{\Sigma}\right) \subseteq$ $\bar{\Delta}^{\Sigma}$. Then there exists an automorphism $\Phi$ of $F_{n}^{\infty}$ such that $\widehat{\Phi}=\phi^{-1}$ and $\Phi\left(\mathcal{N}_{\Delta}\right) \subseteq$ $\mathcal{N}_{\Delta}$, so that $\Phi$ induces an automorphism of $F_{n}^{\infty} / \mathcal{N}_{\Delta}$.

Proof. If $\phi$ is any automorphism of $\mathbb{B}_{n}$ such that $\phi\left(\bar{\Delta}^{\Sigma}\right) \subseteq \bar{\Delta}^{\Sigma}$, then by [3] there exists a unique automoprhism $\Phi$ of $F_{n}^{\infty}$ such that $\phi^{-1}$ is the dual map of $\Phi$ on the spectrum $\mathbb{B}_{n}$ of $F_{n}^{\infty}$. Now, let $x \in \mathcal{N}_{\Delta}$. By construction, if $\lambda \in \bar{\Delta}^{\Sigma}$, then $\Phi(x)(\lambda)=x(\phi(\lambda))=0$ since $\phi(\lambda) \in \bar{\Delta}^{\Sigma}$. Hence $\Phi\left(\mathcal{N}_{\Delta}\right) \subseteq \mathcal{N}_{\Delta}$, and thus $\Phi$ induces an automorphism of $F_{n, \Delta}^{\infty}$.

The converse implication, i.e. that any automorphism of $F_{n}^{\infty} / \mathcal{N}_{\Delta}$ is given by an automorphism of $\mathbb{B}_{n}$ which maps the spectrum to itself, is the subject of the rest of this paper. To this end, we shall use the following special case of [12, Theorem 8.2.2]:

Lemma 2.2. Let $\Phi: \mathbb{B}_{n} \rightarrow \mathbb{B}_{n}$ be a holomorphic map with $\Phi(0)=0$. If the set $\left\{\lambda \in \mathbb{B}_{n}: \Phi(\lambda)=\lambda\right\}$ of fixed points of $\Phi$ spans $\mathbb{C}^{n}$, then $\Phi$ is the identity.

Proof. Our assumption implies the existence of a basis of invariant vectors for the Frechet derivative $\Phi^{\prime}(0)$ of $\Phi$ at 0 , so $\Phi^{\prime}(0)$ is the identity on $\mathbb{C}^{n}$. By [12, Theorem 8.2.2], $\Phi$ has the same invariant points as $\Phi^{\prime}(0)$, hence our lemma.

We now establish the key result for this section. The class of $x \in F_{n}^{\infty}$ in $F_{n, \Delta}^{\infty}$ is denoted by $x+\mathcal{N}_{\Delta}$. To make the proof of Theorem 2.9 clearer, we organize it as a succession of lemmas. The first step is to construct automorphisms of $\mathbb{B}_{n}$ from automorphisms of $F_{n, \Delta}^{\infty}\left(\Delta \subseteq \mathbb{B}_{n}\right)$. 
Lemma 2.3. Let $\Delta \subseteq \mathbb{B}_{n}$ such that $\bar{\Delta}^{\Sigma}$ spans $\mathbb{C}^{n}$. Let $\Phi$ be a completely contractive automorphism of $F_{n, \Delta}^{\infty}$. Then there exists $\varphi_{1}, \ldots, \varphi_{n} \in F_{n}^{\infty}$ such that:

- We have $\left\|\left[\varphi_{1}, \ldots, \varphi_{n}\right]\right\|_{M_{1, n}\left(F_{n}^{\infty}\right)} \leq 1$.

- We have $\left[\Phi^{-1}\left(S_{j}+\mathcal{N}_{\Delta}\right)\right]_{j=1, \ldots, n}=\left[\varphi_{j}\right]_{j=1, \ldots, n}+M_{1, n}\left(\mathcal{N}_{\Delta}\right)$.

- The dual map $\widehat{\Phi}$ of $\Phi$ on the spectrum $\bar{\Delta}^{\Sigma}$ of $F_{n, \Delta}^{\infty}$ is given by $\lambda \mapsto$ $\left(\varphi_{1}(\lambda), \ldots, \varphi_{n}(\lambda)\right)$.

- The map $\overleftrightarrow{\Phi}=\left(\varphi_{1}, \ldots, \varphi_{n}\right)$ is a biholomorphic map from $\mathbb{B}_{n}$ onto $\mathbb{B}_{n}$.

Proof. Let $\Phi$ be an automorphism of $F_{n, \Delta}^{\infty}$. The element

$$
\left[\Phi^{-1}\left(S_{1}+\mathcal{N}_{\Delta}\right) \cdots \Phi^{-1}\left(S_{n}+\mathcal{N}_{\Delta}\right)\right] \in M_{1, n}\left(F_{n, \Delta}^{\infty}\right)=M_{1, n}\left(F_{n}^{\infty}\right) / M_{1, n}\left(\mathcal{N}_{\Delta}\right)
$$

is a row contraction, since $\left[S_{1} \cdots S_{n}\right]$ is as well, and $\Phi$ is completely isometric, hence so is $\Phi^{-1}$. Now, by definition

$$
\begin{aligned}
1 & \geq\left\|\left[\Phi^{-1}\left(S_{1}+\mathcal{N}_{\Delta}\right) \cdots \Phi^{-1}\left(S_{n}+\mathcal{N}_{\Delta}\right)\right]\right\|_{M_{1, n}\left(F_{n, \Delta}^{\infty}\right)} \\
& =\inf _{\substack{\psi_{j}+\mathcal{N}_{\Delta}=\Phi^{-1}\left(S_{j}\right) \\
j=1, \ldots, n}}\left\|\left[\psi_{1} \cdots \psi_{n}\right]\right\|_{M_{1, n}\left(F_{n}^{\infty}\right)} .
\end{aligned}
$$

Since the unit ball of $F_{n}^{\infty}$ is weak* compact, and hence so is the unit ball of $M_{1, n}\left(F_{n}^{\infty}\right)$, and since $\mathcal{N}_{\Delta}$ is weak* closed, we can find $\varphi_{1}, \ldots, \varphi_{n} \in F_{n}^{\infty}$ such that $\left\|\left[\varphi_{1} \cdots \varphi_{n}\right]\right\|_{M_{1, n}}=1$ and

$$
\left[\Phi^{-1}\left(S_{1}+\mathcal{N}_{\Delta}\right) \cdots \Phi^{-1}\left(S_{n}+\mathcal{N}_{\Delta}\right)\right]=\left[\varphi_{1} \cdots \varphi_{n}\right]+M_{1, n}\left(\mathcal{N}_{\Delta}\right) .
$$

Now, let $\widehat{\Phi}$ be the dual map of $\Phi$ on $\bar{\Delta}^{\Sigma}$. Note that in particular $\widehat{\Phi}\left(\bar{\Delta}^{\Sigma}\right) \subseteq$ $\bar{\Delta}^{\Sigma} \subseteq \mathbb{B}_{n}$. Let $\lambda \in \bar{\Delta}^{\Sigma}$. Then

$$
\begin{aligned}
\widehat{\Phi}(\lambda) & =\left(\left[\Phi^{-1}\left(S_{1}+\mathcal{N}_{\Delta}\right)\right](\lambda), \ldots,\left[\Phi^{-1}\left(S_{n}+\mathcal{N}_{\Delta}\right)\right](\lambda)\right) \\
& =\left(\left(\varphi_{1}+\mathcal{N}_{\Delta}\right)(\lambda), \ldots, \varphi_{n}+\mathcal{N}_{\Delta}(\lambda)\right) \\
& =\left(\varphi_{1}(\lambda), \ldots, \varphi_{n}(\lambda)\right)
\end{aligned}
$$

since for all $\theta \in \mathcal{N}_{\Delta}$ we have $\theta(\lambda)=0$. Thus $\overleftrightarrow{\Phi}:=\left(\varphi_{1}, \ldots, \varphi_{n}\right)$, which is a holomorphic map from $\mathbb{B}_{n}$ to $\overline{\mathbb{B}_{n}}$ (since $\left\|\left[\varphi_{1} \cdots \varphi_{n}\right]\right\| \leq 1$ ), is an analytic extension of $\widehat{\Phi}$ to $\mathbb{B}_{n}$. Now, let $z \in \mathbb{B}_{n}$ and suppose that $\overleftrightarrow{\Phi}(z)$ lies on the boundary of $\overline{\mathbb{B}_{n}}$. Then, up to conjugating $\overleftrightarrow{\Phi}$ by a biholomorphic map, we may as well assume that $\overleftrightarrow{\Phi}(0)$ lies on the boundary of $\overline{\mathbb{B}_{n}}$. Again, up to conjugation by a unitary, we may as well assume that $\overleftrightarrow{\Phi}(0)=(1,0, \ldots, 0)$. Thus $\varphi_{1}(0)=1$ and $\varphi_{1}: \mathbb{B}_{1} \rightarrow \overline{\mathbb{B}_{1}}$ is holomorphic. We conclude by the maximum modulus principle that $\varphi_{1}$ is the constant function 1 on $\mathbb{B}_{1}$. Therefore $\varphi_{2}=\cdots=\varphi_{n}=0$. Therefore, $\overleftrightarrow{\Phi}$ maps all of $\mathbb{B}_{n}$ to a constant value on the boundary of $\overline{\mathbb{B}_{n}}$, which contradicts the fact that $\overleftrightarrow{\Phi}\left(\bar{\Delta}^{\Sigma}\right) \subseteq \mathbb{B}_{n}$. Hence, $\overleftrightarrow{\Phi}$ is a holomorphic map from $\mathbb{B}_{n}$ into $\mathbb{B}_{n}$

With the same technique, we can construct a holomorphic map $\overleftrightarrow{\Phi^{-1}}$ from $\mathbb{B}_{n}$ into itself whose restriction to $\bar{\Delta}^{\Sigma}$ is the map $\widehat{\Phi^{-1}}$ dual to the inverse $\Phi^{-1}$ of $\Phi$ on $\bar{\Delta}^{\Sigma}$.

Now, by construction, $\overleftrightarrow{\Phi^{-1}} \circ \overleftrightarrow{\Phi}(\lambda)=\lambda$ for all $\lambda \in \bar{\Delta}^{\Sigma}$ and $\overleftrightarrow{\Phi^{-1}} \circ \overleftrightarrow{\Phi}$ is a holomorphic function from $\mathbb{B}_{n}$ to $\mathbb{B}_{n}$. Since the span of $\bar{\Delta}^{\Sigma}$ is $\mathbb{C}^{n}$, we conclude with 
Lemma 2.2 that $\overleftrightarrow{\Phi^{-1}} \circ \overleftrightarrow{\Phi}$ is the identity of $\mathbb{B}_{n}$. The same exact reasoning shows that $\overleftrightarrow{\Phi} \circ \overleftrightarrow{\Phi^{-1}}$ is also the identity of $\mathbb{B}_{n}$.

This concludes our lemma.

Our next lemma establishes that the map $\Phi \in \operatorname{Aut}\left(F_{n, \Delta}^{\infty}\right) \mapsto \widehat{\Phi}$ which maps an automorphism of $F_{n, \Delta}^{\infty}$ to its dual map on the spectrum of $F_{n, \Delta}^{\infty}$ is in fact a group monomorphism when $\Delta$ spans $\mathbb{C}^{n}$. We briefly recall from [7] the following construction. We abbreviate the notation $F^{2}\left(\mathbb{C}_{n}\right)$ into $F_{n}^{2}$. Given a vector $\xi \in F_{n}^{2}$, we define $\|\xi\|$ as the supremum of $\|\xi \otimes \eta\|_{F_{n}^{2}}$ over a finite linear combination of elementary tensors $\eta$ in $F_{n}^{2}$ with $\|\eta\|_{F_{n}^{2}} \leq 1$. If $\xi \in F_{n}^{2}$ and $\|\xi\|<\infty$, then the operator $\eta \in F_{n}^{2} \mapsto \xi \otimes \eta$, still denoted by $\xi$, is a well-defined linear operator of norm $\|\xi\|$. From [7, we see that $F_{n}^{\infty}=\left\{\xi \in F_{n}^{2}:\|\xi\|<\infty\right\}$ and $\|\cdot\|=\|\cdot\|_{F_{n}^{\infty}}$. We also note that $\|\cdot\|_{F_{n}^{\infty}} \geq\|\cdot\|_{F_{n}^{2}}$. With this identification in mind, we show:

Lemma 2.4. Let $\Delta \subseteq \mathbb{B}_{n}$ such that $\Delta$ spans $\mathbb{C}^{n}$. Let $\Phi$ be a completely contractive automorphism of $F_{n, \Delta}^{\infty}$. If the dual map $\widehat{\Phi}$ of $\Phi$ on the spectrum $\bar{\Delta}^{\Sigma}$ of $F_{n, \Delta}^{\infty}$ is the identity, then $\Phi$ is the identity.

Consequently, $\Phi \in \operatorname{Aut}\left(F_{n, \Delta}^{\infty}\right) \mapsto \widehat{\Phi}$, which maps an automorphism of $F_{n, \Delta}^{\infty}$ to its dual map, is a group monomorphism.

Proof. By Lemma 2.3 there exists $\varphi_{1}, \ldots, \varphi_{n} \in F_{n}^{\infty}$ such that

$$
\left[\Phi^{-1}\left(S_{j}+\mathcal{N}_{\Delta}\right)\right]_{j=1, \ldots, n}=\left[\varphi_{j}\right]_{j=1, \ldots, n}+M_{1, n}\left(\mathcal{N}_{\Delta}\right)
$$

and $\left\|\left[\varphi_{j}\right]_{j=1 \ldots n}\right\|_{M_{1, n}} \leq 1$. Now, $\overleftrightarrow{\Phi}=\left(\varphi_{1}, \ldots, \varphi_{n}\right)$ fixes $\bar{\Delta}^{\Sigma}$ by assumption, so $\overleftrightarrow{\Phi}$ is the identity by Lemma 2.2. Therefore, $\varphi_{j}=S_{j}+C_{j}$ for some $C_{j} \in F_{n}^{\infty}$ such that $C_{j}(\lambda)=0$ for all $\lambda \in \mathbb{B}_{n}$, where $j=1, \ldots, n$. Hence $C_{j}$ lies in the ideal of $F_{n}^{\infty}$ generated by $S_{j} S_{k}-S_{k} S_{j}(k, j=1, \ldots, n)$.

Let $j \in\{1, \ldots, n\}$. Let $e_{j}$ be the $j^{\text {th }}$ canonical basis vector in $\mathbb{C}^{n}$ and let $\xi_{j} \in F_{n}^{2}$ such that $C_{j}(\eta)=\xi_{j} \otimes \eta$ for all $\eta \in F_{2}^{n}$. Then $\xi_{j}$ is orthogonal to $e_{j}$ in $F_{2}^{n}$ and therefore

$$
1 \geq\left\|\varphi_{j}\right\|_{F_{n}^{\infty}}^{2} \geq\left\|e_{j}+\xi_{j}\right\|_{F_{n}^{2}}^{2}=\sqrt[2]{\left\|e_{j}\right\|_{F_{n}^{2}}+\left\|\xi_{j}\right\|_{F_{n}^{2}}^{2}} .
$$

Since $\left\|e_{j}\right\|=1$ we conclude $\xi_{j}=0$, and thus $C_{j}=0$. Hence $\Phi^{-1}\left(S_{j}+\mathcal{N}_{\Delta}\right)=$ $S_{j}+\mathcal{N}_{\Delta}$, so $\Phi$ is the identity on $F_{n . \Delta}^{\infty}$, as desired.

In particular, the kernel of $\Phi \in \operatorname{Aut}\left(F_{n, \Delta}^{\infty}\right) \mapsto \widehat{\Phi}$ is reduced to the identity, so this group homomorphism is injective.

Theorem 2.5. Let $\Delta \subseteq \mathbb{B}_{n}$ and let $\Gamma$ be the subgroup of $\mathrm{SU}(n, 1)$ such that $\gamma \in \Gamma$ if and only if $\gamma\left(\bar{\Delta}^{\Sigma}\right)=\bar{\Delta}^{\Sigma}$. If the span of $\bar{\Delta}^{\Sigma}$ is $\mathbb{C}^{n}$, then the automorphism group of $F_{n, \Delta}^{\infty}$ is $\Gamma$.

Proof. Let $\Phi$ be an automorphism of $F_{n, \Delta}^{\infty}$. By Lemma 2.3, we can extend the dual map $\widehat{\Phi}$ on the spectrum $\bar{\Delta}^{\Sigma}$ of $F_{n, \Delta}^{\infty}$ into an automorphism $\overleftrightarrow{\Phi}$ of $\mathbb{B}_{n}$ which maps $\bar{\Delta}^{\Sigma}$ onto itself. By Lemma 2.4, the map $\Phi \in \operatorname{Aut}\left(F_{n, \Delta}^{\infty}\right) \mapsto \widehat{\Phi}$ is a group monomorphism into the group of homeomorphism of $\bar{\Delta}^{\Sigma}$. Now, by Lemma 2.2, the map $\widehat{\Phi} \mapsto \overleftrightarrow{\Phi}$ constructed in Lemma 2.3 is also a group monomorphism. Indeed, if any two automorphisms of $\mathbb{B}_{n}$ agree on $\bar{\Delta}^{\Sigma}$, then they must be equal. Since $\overleftrightarrow{\Phi_{1}} \circ \overleftrightarrow{\Phi_{2}}$ 
certainly restricts on $\bar{\Delta}^{\Sigma}$ to $\widehat{\Phi_{1}} \circ \widehat{\Phi_{2}}=\widehat{\Phi_{1} \circ \Phi_{2}}$, we conclude by uniqueness that $\overleftrightarrow{\Phi_{1}} \circ \overleftrightarrow{\Phi_{2}}=\overleftrightarrow{\Phi_{1} \circ \Phi_{2}}$ for any $\Phi_{1}, \Phi_{2} \in \operatorname{Aut}\left(F_{n, \Delta}^{\infty}\right)$. Hence, the map $\Phi \in \operatorname{Aut}\left(F_{n, \Delta}^{\infty}\right) \mapsto$ $\overleftrightarrow{\Phi} \in \operatorname{Aut}\left(\mathbb{B}_{n}\right)$ is a group monomorphism. Moreover, by Lemma 2.3, its range is included in the set of automorphisms of $\mathbb{B}_{n}$ which maps $\bar{\Delta}^{\Sigma}$ to itself. The reverse inclusion is established by Lemma 2.1 .

Thus, the map $\Phi \in \operatorname{Aut}\left(F_{n, \Delta}^{\infty}\right) \longmapsto \overleftrightarrow{\Phi} \in \operatorname{Aut}\left(\mathbb{B}_{n}\right)$ is a well-defined group isomorphism. This concludes the computation of the automorphism group of $F_{n, \Delta}^{\infty}$.

Remark 2.6. As a by-product of the proof above, we see that any automorphism of $F_{n, \Delta}^{\infty}$ lifts uniquely to an automorphism of $F_{n}^{\infty}$. Even more, the group of automorphisms of $F_{n, \Delta}^{\infty}$ is the quotient of the group of automorphisms of $F_{n}^{\infty}$ by the stabilizer subgroup of $\mathcal{N}_{\Delta}$.

We conclude this section with the main result of this paper. Given a discrete subgroup $\Gamma$ of $\mathrm{SU}(n, 1)$, when is the automorphism group of a quotient of $F_{n}^{\infty}$ isomorphic to $\Gamma$ ? In general, it is not true that the automorphism group of $F_{n, \Gamma(0)}^{\infty}$ is $\Gamma$, as shown in the following example.

Example 2.7. Let $\Gamma=\left\{\gamma^{n}: n \in \mathbb{Z}\right\}$ where $\gamma: z \in \mathbb{B}_{1} \mapsto \frac{z-\frac{1}{2}}{1-\frac{1}{2} z}$. Then the rotation of center 0 and angle $\pi$ is an (elliptic) automorphism of $\mathbb{B}_{n}$ which maps the orbit $\Gamma(0)$ of 0 for $\Gamma$ to itself. Thus by Proposition 2.5, it is an automorphism of $F_{n, \Delta}^{\infty}$. Yet it is not an element of $\Gamma$, which only consists of hyperbolic automorphisms.

In sight of Example 2.7 it is only natural to define:

Definition 2.8. Let $\Gamma$ be a subgroup of $\mathrm{SU}(n, 1)$. Let $\underline{\Gamma}$ be the stabilizer subgroup of the orbit $\Gamma(0)$ of 0 by $\Gamma$ in $\mathrm{SU}(n, 1)$, i.e.,

$$
\underline{\Gamma}=\{\gamma \in \mathrm{SU}(n, 1): \forall z \in \Gamma(0) \quad \gamma(z) \in \Gamma(0)\} .
$$

Example 2.7 shows that $\Gamma$ may be a strict subgroup of $\underline{\Gamma}$.

We can now put our results together to show that:

Theorem 2.9. Let $\Gamma$ be a discrete subgroup of $\mathrm{SU}(n, 1)$ such that

$$
\sum_{\gamma \in \Gamma}\left(1-\|\gamma(0)\|_{\mathbb{C}^{n}}\right)<\infty
$$

and the orbit $\Gamma(0)$ of 0 spans $\mathbb{C}^{n}$. Then the automorphism group of $F_{n, \Gamma(0)}^{\infty}=$ $F_{n}^{\infty} / \mathcal{N}_{\Gamma(0)}$ is the stabilizer subgroup $\underline{\Gamma}$ in $\mathrm{SU}(n, 1)$ of $\Gamma(0)$. Moreover, the action of $\Gamma$ on $F_{n, \Gamma(0)}^{\infty}$ is ergodic.

Proof. By Theorem 1.11, the spectrum of $F_{n, \Gamma(0)}^{\infty}$ is $\Gamma(0)$. By Theorem 2.5, the group of automorphisms of $F_{n, \Gamma(0)}^{\infty}$ is $\underline{\Gamma}$. To ease notation, let $\tau$ be the group isomorphism from $\underline{\Gamma}$ onto Aut $\left(F_{n, \Delta}^{\infty}\right)$ given by Theorem 2.5 .

Now let $a \in F_{n, \Gamma(0)}^{\infty}$ such that for all $\gamma \in \Gamma$ we have $\tau(\gamma)(a)=a$, so $\lambda \in$ $\Gamma(0) \mapsto a(\lambda)$ is constant, equal to $a(0)$. Hence $a-a(0) 1$ is 0 on $\Gamma(0)$, and thus $\varphi-a(0) 1 \in \mathcal{N}_{\Gamma(0)}$, i.e., $a=\varphi+\mathcal{N}_{\Gamma(0)}=a(0) 1$ is a scalar multiple of the identity. So $\Gamma$ acts ergodically. 
We can deduce from Theorem 2.9 the following simple corollary, where the span condition is relaxed, but the relation between the original group and the automorphism group may be less clear.

Corollary 2.10. Let $\Gamma$ be a a discrete subgroup of $\mathrm{SU}(n, 1)$ such that

$$
\sum_{\gamma \in \Gamma}\left(1-\|\gamma(0)\|_{\mathbb{C}^{n}}\right)<\infty
$$

and the orbit $\Gamma(0)$ of 0 spans $\mathbb{C}^{k}$ with $k \leq n$. Then the automorphism group of $F_{n, \Gamma(0)}^{\infty}=F_{n}^{\infty} / \mathcal{N}_{\Gamma(0)}$ is isomorphic to the stabilizer subgroup $\underline{\Gamma}$ in $\mathrm{SU}(k, 1)$ of the image of $\Gamma(0)$ by a unitary $U$ of $\mathbb{C}^{n}$ such that $\operatorname{Ad} U(\Gamma(0)) \subseteq \mathbb{C}^{k} \times\{0\}$.

Proof. Let $U$ be a unitary acting on $\mathbb{C}^{n}$ such that $\Delta=U \Gamma(0) U^{*} \subseteq \mathbb{C}^{k} \times\{0\}$. Hence, the span of $\Delta$ is $\mathbb{C}^{k} \times\{0\}$. Now, write $\mathfrak{U}$ for the automorphism of $F_{n}^{\infty}$ such that $\widehat{\mathfrak{U}}=\operatorname{Ad} U$. Now $\mathfrak{U}$ maps $\mathcal{N}_{\Gamma(0)}$ onto $\mathcal{N}_{\Delta}$ and thus defines an isomorphism from $F_{n, \Gamma(0)}^{\infty}$ onto $F_{n, \Delta}^{\infty}$ still denoted by $\mathfrak{U}$. It is now easy to see that the quotient map $F_{n}^{\infty} \longrightarrow F_{n, \Delta}^{\infty}$ factors as $F_{n}^{\infty} \longrightarrow F_{k}^{\infty} \longrightarrow F_{n, \Delta}^{\infty}=F_{k, \Delta}^{\infty}$. Now by Theorem 2.5, the group of automorphisms of $F_{n, \Delta}^{\infty}$ is the stabilizer group $\underline{\Gamma}$ of $\Delta$ in $\operatorname{SU}(k, 1)$.

Last, we note that $\Phi \in \operatorname{Aut}\left(F_{n, \Gamma(0)}^{\infty}\right) \mapsto \mathfrak{U} \circ \Phi \circ \mathfrak{U}^{-1}$ is a group isomorphism onto $\operatorname{Aut}\left(F_{n, \Delta}^{\infty}\right)$.

We conclude that, using methods similar to the proof of Theorem 2.5] we can also prove that:

Corollary 2.11. Let $\Delta_{1}$ and $\Delta_{2}$ be two subsets of $\mathbb{B}_{n}$. Then there exists a completely isometric isomorphic from $F_{n, \Delta_{1}}^{\infty}$ to $F_{n, \Delta_{2}}^{\infty}$ if and only if there exists an automorphism $\gamma \in \mathrm{SU}(n, 1)$ of $\mathbb{B}_{n}$ such that $\gamma\left({\overline{\Delta_{1}}}^{\Sigma}\right)={\overline{\Delta_{2}}}^{\Sigma}$.

\section{REFERENCES}

1. A. Arias and F. Latrémoliere, Isomorphisms of non-commutative domain algebras, to appear in J. Oper. Theory.

2. W. Arveson, Subalgebras of $C^{*}$-algebras. III. Multivariable operator theory, Acta Math. 181 (1998), no. 2, 159-228. MR1668582 (2000e:47013)

3. K. Davidson and D. Pitts, The algebraic structure of non-commutative analytic Toeplitz algebras, Math. Ann. 311 (1998), 275-303. MR.1625750 (2001c:47082)

4. _ Invariant subspaces and hyper-reflexivity for free semigroup algebras, Proc. London Math. Soc. (3) 78 (1999), no. 2, 401-430. MR1665248 (2000k:47005)

5. S. Krantz, Function theory of several complex variables, Mathematics Series, Wadsworth and Brooks/Cole, 1992.

6. _ Handbook of complex variables, Birkhäuser, Boston, MA, 1999. MR.1738432 (2001a:30001)

7. G. Popescu, Von Neumann inequality for $\left(B(\mathcal{H})^{n}\right)_{1}$, Math. Scand. 68 (1991), no. 2, 292-304. MR:1129595 (92k:47073)

8. , Non-commutative disc algebras and their representations, Proc. Amer. Math. Soc. 124 (1996), no. 7, 2137-2148. MR1343719 (96k:47077)

9. _ Poisson transforms on some $C^{*}$-algebras generated by isometries, J. Funct. Anal. 161 (1999), no. 1, 27-61. MR.1670202 (2000m:46117)

10. - Operator theory on noncommutative domains, Mem. Amer. Math. Soc. 205 (2010), no. 964 , vi +124 pp.

11. _ Free holomorphic automorphisms of the unit ball of $B(\mathcal{H})^{n}$, J. Reine Angew. Math. 638 (2010), 119-168. MR2595338 
12. W. Rudin, Function theory in the unit ball of $\mathbf{C}^{n}$, Grundlehren der Mathematischen Wissenschaften, vol. 241, Springer-Verlag, 1980. MR0601594 (82i:32002)

13. D. Voiculescu, Symmetries of some reduced free product $C^{*}$-algebras, Lecture Notes in Mathematics, vol. 1132, Springer-Verlag, Berlin, 1985. MR799593 (87d:46075)

Department of Mathematics, University of Denver, Denver, Colorado 80208

E-mail address: aarias@math.du.edu

$U R L:$ http://www.math.du.edu/ aarias

Department of Mathematics, University of Denver, Denver, Colorado 80208

E-mail address: frederic@math.du.edu

URL: http://www.math.du.edu/ ${ }^{\sim}$ rederic 